und so ist das Erscheinen der Lebensmitteltechnologie von R. HeIss sehr zu begrüßen. Im 1. Abschnitt werden die verfahrenstechnisehen Möglichkeiten, die Ảnderung der Stoffdichte, die Mischvorgänge, Stofftrennung usw. besprochen. Im 2. Abschnitt folgen nach gleichen Gesichtspunkten angeordnet die mechanischen Herstellungsverfahren, im 3. Abschnitt Verfahren, bei denen thermische Prozesse überwiegen. Biochemische Verfahren bilden den letzten Abschnitt. Aus dieser Einteilung ergibt sich, daß das gleiche Lebensmittel wie z. B. Fett, Getreide, Milch, entsprechend der jeweiligen technischen Bearbeitung sich in verschiedenen $\mathrm{Ab}$ schnitten findet. Durch das gute Sachverzeichnis entstehen hierdurch jedoch keinerlei Schwierigkeiten. Der klare Text wird durch eine große Zahl von Abbildungen ausgezeichnet ergänzt. Die Herstellungsprozesse sind vielfach übersichtlich durch Schemen wiedergegeben. Da alle wichtigen Lebensmittel berücksichtigt sind, ist nur eine gedrängte Wiedergabe der wesentlichen Tatsachen möglich, besondere Probleme bleiben Sache der Spezialwerke. Die Heranziehung des Buches bei der täglichen Arbeit hat bereits in kurzer Zeit dem Referenten wertvolle Dienste erwiesen und so ist es zu erwarten, daß dieses Werk sicher bald in der Hand aller sein wird, die sich mit Lebensmittelchemie und Technologie befassen.

\title{
W. Freseñus.
}

Zur titrimetrisehen Bestimmung des übersehüssigen Natriumearbonats in Backpulvern legen H. HadorN und K. W. BiEFER ${ }^{1}$ eine eigene Arbeitsvorschrift vor, da die im Schweiz. Lebensmittelbuch angegebene Methode zu unrichtigen Ergebnissen führen soll.

2-4 g Backpulver werden in einem mit Uhrglas bedeckten Becherglas mit etwa $400 \mathrm{ml}$ Wasser aufgeschlämmt und mit $20 \mathrm{ml} 0,5 \mathrm{n}$ Salzsäure versetzt. Unter Rühren mit einem Glasstab wird so lange gekocht, bis alle Kohlensäure ausgetrieben ist. Die heiße Lösung wird mit 0,5 n Natronlauge mit Phenolphthalein als Indikator auf schwach rosa titriert. Der. Verbrauch an Säure entspricht dem Überschuß an Natriumbicarbonat. - Bei Anwesenheit von Pyrophosphaten wird in der Kälte mit Thymolphthalein auf erste Blaufärbung titriert.

L. ACKER.

Ascorbinsäure. E. GERoª̈berprüfte einige Methoden zur Bestimmung der Ascorbinsäure. Er setzte Extrakten aus tierischen Organen und vegetabilischen Stoffen bestimmte Mengen an Vitamin $\mathrm{C}$ zu und bestimmte die zugesetzte Menge nach 3 Verfahren: Mit der Methode von Tillmans wurden die besten Ergebnisse erzielt, während die kinetische Methode von Meunren ${ }^{3}$, bei der aus der Kinetik der Entfärbung von Dichlorphenol-indophenol der Vitamingehalt errechnet wird, gelegentlich sehr schwankende Ergebnisse liefert. Auch die Methylenblaureduktion gibt keine zuverlässigen Werte. In seinen umfangreichen Untersuchungen konnte der Verf. zeigen, daß die Schwankungen der beiden letzten Methođen auf Trübungen zurückzuführen sind, die einmal an sich die photometrische Auswertung exschweren, zum andern aber auch einen Teil des Farbstoffs adsorbieren, der sich dadurch der Messung entzieht. Nach längerem Zentrifugieren konnte tatsächlich ein gefärbter Rückstand erhalten werden.

1 Mitt. Lebensmittelunters. 10,352 (1949).

2 Bull. Soc. Chim. biol. Paris 31, 817 (1949).

3 Meunter P. und Y. Raodl, Le diagnostic chimique des avitaminoses. 1942. Masson, Paris. 